Pub. Mat. UAB

Vol. 30 no 1 Maig 1986

\title{
ON HOMOGENITY AND TRANSITIVITY \\ OF FIELDS OF GEOMETRIC OBJECTS
}

Jerzy Konderak

ABSTRACT. If $\sigma$ is a field of geometric objects on a manifold $M$ then we can associate with it a principal subbundle of $\mathrm{H}^{r}(\mathrm{M})$. We show that (infinitesimal) homogenity and (infinitesi mal) transitivity of this subbundle are equivalent to some inte gral conditions for the lie eouations generated by $o$.

\section{INTRODUCTION}

1. Let $M$ be a differentiable manifold. In the present paper, manifolds, vector fields and so on always mean differentiable manifolds, differentiable vector fields and so on. Diffe rentiability always means the differentiability of class $\mathrm{C}^{\infty}$.

If $U, V$ are open subsets of $M$ then a diffeomerphism $\varphi: U \longrightarrow V$ is called a lccal diffeomorphism of $M$. The set $\Gamma(M)$ of all local diffeomorphisms of $M$ is a pseudogroup. By TI: we shall denote a set of vector fields defined on open subsets of $M$.

We denote by $\mathrm{H}^{\mathrm{r}}$ (M) the set of all r jets at 0 of didiffeomorphisms of open neighbourhoods of 0 in $\mathbb{R}^{\mathrm{n}}$ onto open subsets of $M$. Let $\pi^{r}: H^{r}(M) \longrightarrow M$ be the target projection. Then $\mathrm{H}^{r}(M)$ is a principal fibre burdle with the 
structure group $\mathrm{L}_{\mathrm{n}}^{\mathrm{x}}$ of all r-jets with the source and with the target at 0 of local diffeomorphisms of $\operatorname{IR}^{n}$.

2. Let $F$ denote a natural bundle from the category of n-dimensional manifolds (for the definition of the natural bundle see [7] or [8]). Differentiable sections of the bundle $\pi: F(M) \longrightarrow M$ are called fields of geometric objects. Ve assume that $F$ is of order $r$. It means that if $U, V$ are $n-d i m e n-$ sional manifolds and $\varphi, \psi: U \longrightarrow V$ are diffeomorphisms such that $j_{x}^{r} \varphi=j_{x}^{r} \psi$ for a certain $x \in U$ then $F(\varphi)_{\pi}-l_{(x)}=$ $=F(\psi)_{\pi^{-1}}$, that is $F(\varphi)$ and $F(\psi)$ are equal on the $f i b e r$ of $\pi: F(U) \longrightarrow U$ above the point $x$. Let $F_{0}=\pi^{-1}(0)$. where $\pi: F\left(\mathbb{R}^{n}\right) \rightarrow \mathbb{R}^{n}$, be called a standard fibre of the hundle $\mathbf{F}$.

If $x \in T M$ then the flow of $x$ induces a flow on $F(\operatorname{dom}(x))$. The vector field on F(dom (X)) defined by this flow is called a complet lift of $x$ and is usually denoted by $F(x)$ (cf. [9]).

A functor $\mathrm{H}^{\mathrm{r}}$ which attaches to each n-aimensional mani fold $M$ the principal fibre bundle $H^{r}(M)$ is an example of a natural bundle. If $\varphi: \mathrm{N}_{1} \longrightarrow \mathrm{N}_{2}$ is an embedding of $n$-dimensional manitolds then $\mathrm{H}^{\mathrm{r}}(\varphi)\left(\mathrm{j}_{0}^{\mathrm{r}} \mathrm{f}\right)=j_{0}^{r}(\varphi, f)$ where $j_{0}^{\mathrm{r}} \mathrm{f} \in \mathrm{H}^{\mathrm{r}}\left(\mathrm{N}_{1}\right)$. The bundle $\mathrm{H}^{\mathbf{r}}$ is of course of order $r$. A complet lift of $\mathrm{X} \in \underline{\mathrm{TM}}$ to the bundle $\mathrm{H}^{x}(\mathrm{M})$ will be denoted by $H^{\mathrm{r}}(\mathrm{X})$. The concept of 'natural bundle' was introduced by A. Nijenhuis ([7]) as a modern approach to the classical theory of geometric objects (cf. [1]).

Let $\sigma$ be a fiel of geometric objects on $M$. Then $\sigma$ 
induces a mapping $\tau_{\sigma}: H^{Y}(M) \rightarrow F_{0}$ such that

$$
r_{\sigma}\left(j_{0}^{r} f\right)=E\left(f^{-1}\right) \sigma(f(0))
$$

Let $\xi_{0} \mathrm{Fim}_{0} \tau_{0}$. We assume that the set $E=\left(\tau_{0}\right)^{-1}\left(\xi_{0}\right)$ is a principal subbundle of $H^{2}(M)$. It is easy to see that this is equivalent to the following fact: for each $x, y \in M$ there exists $\varphi \in \Gamma(M)$ such that $F(\varphi) \sigma(x)=\sigma(y)$. In the other words one can say that $\sigma$ is o-deformable (cf. [10]). Concerning fields of geonetric objects one usually assumes that they are 0 -deformable. The bundle $E$ is not uniquely determited by $\sigma$ and it also depends on the choice of an element of $\xi E_{0}$. Although we fix the bundle $E$, all the results are true for all subbundles of $\mathrm{H}^{\mathrm{r}}(\mathrm{M})$ induced by $a$.

II. LIE EQUATIONS ASSOCIATED WITH THE FIELD OF GEOMETPIC OBJECTS. We shall recali some facts from the theory of lie equations. These facts will be applied in the third part of this paper. The basic definitions of the thoery of lie equations one can find in [2], [3], [4].

1. There are given the natural bundle $F$ of order $r$ and the field of geometric objects $\theta$ on $M$ (cf. I.3). Let

$$
\Pi^{Y}(M)=\left\{\mathrm{j}_{\mathrm{x}}^{r} \varphi: \varphi \in \Gamma(M) \text { and } \mathrm{x} \in \text { don } \varphi\right\} .
$$

Then $\Pi^{r}(M)$ is a tie groupoid (cf. $[6]$ ). Let now

$$
\Pi^{r}(\sigma)=\left\{j_{x}{ }_{\varphi} \in \Pi^{r}(M): F(\varphi) \sigma(x)=\sigma(\varphi(x))\right\}
$$


With the field $\sigma$ we associated also the bundle $E$.

Lemma II.1. The following equality holds

$$
\Pi^{r}(\sigma)=\left\{p^{\prime} \cdot p^{-1}: p, p^{\prime} \in E\right\}
$$

where $\left(j_{0}^{r} g\right) \cdot\left(j_{0}^{r}\right)^{-1}=j_{x}^{r}\left(g \cdot f^{-1}\right)$ for $j_{0}^{r}, j_{0}^{r} g \in H^{r}(M)$ and $x=f(0)$.

Proof. Let $j_{x}^{r} \in \Pi^{r}(0)$ and $y=\varphi(x)$. Let $j_{0}^{x} \in E_{x}$ then $\tau_{g}\left(j_{0}^{r}(\varphi \cdot f)\right)=F\left((\varphi \cdot f)^{-1}\right) \circ(y)=F\left(f^{-1}\right) F\left(\varphi^{-1}\right) \sigma(y)=$ $=F\left(f^{-I} f \sigma(x)=\xi\right.$. so $j_{0}^{r}(\varphi \cdot f) \in E$. Hence we get that $j_{x}^{r}=j_{0}^{r}(\varphi \cdot f) \cdot\left(j_{0}^{r}\right)^{-1}$ and both jets on the right side of this equation belong to $E$. Since then $j_{0}^{r} \in\left\{p^{\prime} \cdot p^{-1}: p^{*}, p \in E\right\}$. Let now $j_{x}^{r} \psi=j_{x}^{r}\left(h \cdot g^{-1}\right)$ where $j_{0}^{r} h, j_{0}^{r} g \in E$. Then $F(\psi) \sigma(x)=F\left(h \circ g^{-1}\right) \sigma(x)=F(h) F\left(g^{-1}\right) \sigma(g(0))=F(h) \tau_{0}\left(j_{0}^{r} g\right)=$ $=F(h) \xi_{0}=F(h) T_{\sigma}\left(j_{0}^{Y} h\right)=F(h) F\left(h^{-1}\right) \sigma(h(0))=\sigma(h(0))$.

Hence we get that $F(\psi) \sigma(x)=\sigma(\psi(x))$.

Therefore by $\{6\}$ we get that $\Pi^{r}(\sigma)$ is a Lie groupoid because it is associated with the bundle $E$.

A local diffeomorphism $\varphi$ is called a local solution of the non-linear Lie equation $\Pi^{\mathrm{r}}(\sigma)$ iff for each $x \in$ dom $\varphi$ $\dot{I}_{x}^{r} \in \Pi^{r}(\sigma) ; \Pi^{r}(\sigma)$ is called completely integrable iff for each $\eta \in \Pi^{r}(\sigma)$ there exists a local solution $\varphi$ such that $\dot{j}_{x}^{r}=\eta$ for a certain $x \in \operatorname{dom} \varphi$.

2. Let now $R^{r}(\sigma)=\left\{j_{x}^{r} x: x \in I_{M}, x \in \operatorname{dom} x\right.$ and $\left.\left(I_{x} \alpha\right)_{x}=0\right\}$. A. Zajtz proved that $R^{r}(0)$ is a linear lie equation and the 
canonical projection pr $: R^{r}(\sigma) \rightarrow$ Ti is surjective where $p r\left(j_{x}^{r} x\right)=x_{x}$. Here $L_{x^{\sigma}}$ denotes a Lie derivative for a field of geonetric objects (cf. [9], [10]).

A vector field $x \in T M$ is called a local solution of $\mathrm{R}^{\mathrm{r}}(\sigma)$ iff for each $\mathrm{x} \in \operatorname{dom}(\mathrm{x})$ we have $\mathrm{j}_{\mathrm{x}}^{\mathrm{r}} \in \mathrm{R}^{\mathrm{r}}(\sigma)$. The $1 \mathrm{i}-$ near Lie equation $\mathrm{R}^{\mathrm{r}}(0)$ is called completely integrable iff for each $\eta \in \mathrm{R}^{r}(\sigma)$ there exists a local solution $\mathrm{x}$ such that $j_{x}^{r} x=\eta$ for a certain $x \in \operatorname{dom}(x)$.

Lemma II.2. The following conditions are eguivalent:

i). $\left(L_{\mathrm{x}} 0\right)_{\mathrm{x}}=0$;

ii). $H^{r}(X)_{z} \in T_{z} E$ where $z \in E_{X}$ and $H^{r}(X)$ is a complete lift of the vector field $x$ to the bundle $H^{r}(M)$.

proof. We have the following canonical mapping $\Phi: H^{r}(M) \rightarrow F(M)$, where $\Phi\left(j_{0}^{r} f\right)=F(E) \xi_{0}$ for $j_{0}^{r} f \in H^{2}(M)$. It was shown that $\Phi$ is a differentiable fibre mapping covering the identity mapping on M. (cf. [10]). It is easy to see that $\Phi^{-1}(\sigma(M))=E$. Hence $T E=(d \Phi)^{-1}(T \sigma(M))$. Let us also remark that if $Z$ is a vector field on $M$ then $H^{r}(Z)$ is projectable on $F(z)$ via $\Phi$.

Let first assume that $\left(I_{x}\right)_{x}=0$. It means that. $d_{x} \sigma\left(x_{x}\right)=F(x)_{\sigma(x)}$ and $F(x)_{\sigma(x)} \in T_{\sigma(x)} \sigma(M)$. Wie have also that $\mathrm{d}_{z} \Phi\left(H^{\Gamma}(\mathrm{X})_{z}\right)=F(X)_{\sigma(x)}$. Hence $H^{r}(X) \in T_{z} E$ and the impli cation i). $\Rightarrow$ ii). is proved.

Let now assume that $H^{\Gamma}(X)_{z} \in T_{z} E$. Therefore $d_{z} \Phi\left(H^{r}(X)_{z}\right) \in T_{\sigma(x)} \sigma(M)$ and moreover $F(X)_{\sigma(x)} \in T_{\sigma(x)} \sigma(M)$. 
Let us notice that the canonical projection $\mathrm{d}_{\sigma(x)}{ }^{\pi}$ :

$: \mathrm{T}_{\sigma(\mathrm{x})} \sigma(\mathrm{M}) \longrightarrow \mathrm{T}_{\mathrm{X}}^{\mathrm{M}}$ is an isomorphism and $\mathrm{X}_{\mathrm{x}}=\mathrm{d}_{\sigma(\mathrm{x})}{ }^{\pi(F(\mathrm{X})} \sigma(\mathrm{x})=$ $d_{\sigma(x)} \pi\left(a_{x} \sigma\left(x_{x}\right)\right)$. Hence $f(x)_{\sigma(x)}=d_{x} \sigma\left(x_{x}\right)$ and this ends the proof of the implication ii). $\Rightarrow$ i).

III. HOMOGENITY AND TRANSITIVITY OF FIELDS OF GEOMETRIC OBJECTS.

In this section we suggest notions of homogenity and transitivity for fields of geometric objects. We also do this in the infinitesimal case. Then we relate this notions to the similar properties of $\mathrm{E}$.

1. We shall use the following notation:

$$
\begin{aligned}
A(\sigma) & =\{\varphi \in \Gamma(M): x \in d o m \varphi \Rightarrow F(\varphi) \sigma(x)=\sigma(\varphi(x))\} \\
(\sigma) & =\left\{x \in \underline{T M}: \varphi_{t} \in A(\sigma) \text { where } \varphi_{t} \text { is the flow of } X\right\} .
\end{aligned}
$$

Definition III.l. We shall call $\sigma$ homogenous iff $A(\sigma)$ acts transitively on $M$ and infinitesimally homogenous iff for every $x \in M$ and $v \in T_{x} M$ there exists $x \in A(\sigma)$ such that $x_{x}=v$.

Definition III.2. We shall call o transitive iff for each $\eta \in \Pi^{r}(\dot{\sigma})$ there exists $\varphi \in A(\sigma)$ such that ${ }_{j}^{r} \varphi=\eta$ where $x$ is a source of $\eta$. We shall call $\sigma$ infinitesimally transitive iff for each $\zeta \in \mathrm{R}^{2}(\sigma)$ there exists $\mathrm{X} \in A(\sigma)$ such that $j_{y}^{r} x=\zeta$ where $y$ is a source of $\zeta$.

The sets $A(\sigma)$ and $A(\sigma)$ can be described in the follo wig. way: 
Lemma III.3. If $a$ is a 0 -deformable field of geometric objects then
a). $A(\sigma)$ is a set of solutions of $\Pi^{r}(\sigma)$;
b). $A(\sigma)$ is a set of solutions of $\mathrm{R}^{\mathrm{r}}(\sigma)$.

Proof: If $\varphi \in \Gamma(M)$ then $\varphi$ is a local solution of $\Pi^{r}(\sigma)$ iff for each $x \in \operatorname{dom} \varphi j_{x}^{r} \in \Pi^{r}(\sigma)$. This is equivalent to the fact that for each $x \in \operatorname{dom} \varphi \mathrm{F}(\varphi) \sigma(x)=\sigma(\varphi(x))$. Hence $\varphi$ is a local solution of $\boldsymbol{\Pi}^{\mathrm{r}}(\sigma)$ iff $\varphi \in A(\sigma)$ so a). is proved.

Let $x \in A(\sigma)$ and $x \in$ dom $x$ and let $\varphi_{t}$ denote the flow of $x$ then $F\left(\varphi_{t}\right) \sigma(x)=\sigma\left(\varphi_{t}(x)\right)$. Hence $F(x)_{0(x)}=$ $\mathrm{d}_{\mathrm{x}} \sigma\left(\mathrm{x}_{\mathrm{x}}\right)$. This means that $\left(\mathrm{L}_{\mathrm{x}}\right)_{\mathrm{x}}=0$ because the Lie derivati ve of the field of geometric objects can be expressed in the following way $\left(I_{x^{\sigma}}\right)_{x}=a_{x}^{\sigma}\left(x_{x}\right)-F(x)_{\sigma(x)}$ (cf. [9], [ 10]). Therefore $x$ is a solution of $\mathrm{R}^{r}(0)$.

Let now $Y$ be a solution of $R^{r}(a)$ then it generates the field $F(Y)$ on $F(M)$. Since for every $x \in \operatorname{dom} Y F(Y)_{\sigma}(x)=$ $=\mathrm{d}_{\mathrm{X}} \sigma\left(\mathrm{Y}_{\mathrm{X}}\right)$ then $F(\mathrm{Y})_{J_{\sigma}(\mathrm{dom} Y)}$ is a vector field on $\sigma(\mathrm{dom} Y)$. If $\psi_{t}$, is a flow of $Y$ then $F\left(\psi_{t}\right) \sigma(x) \in$ oldomy). Hence $F\left(\psi_{t}\right) \sigma(x)=\sigma\left(\psi_{t}(x)\right)$ and $Y \in A(\sigma)$. This ends the proof of $\left.b\right)$. From lenua III.3 we get immediatly the following coro1 laxy

Corollary III.4.

i). The field $\sigma$ is homogenous iff for each $x, y \in M$ there exists $\varphi$ a local solution of $\Pi^{r}(\sigma)$ such that $\varphi(x)=y$; 
ii). $\sigma$ is infinitesimally homogenous iff for each

$x \in M$ and $V \in T_{X} M$ there exists $X$ a local solution of $R^{r}(\sigma)$ such that $\mathrm{x}_{\mathrm{x}}=\mathrm{v}$;

iii). $\sigma$ is transitive iff $\mathrm{II}^{\mathrm{r}}(\sigma)$ is completely integrable;

iv). $\sigma$ is infinitesimally transitive iff $\mathrm{R}^{r}(\sigma)$ is completely integrable.

2. With the principal fibre bundle $E$ we can associate the following sets:

$$
\begin{aligned}
& A(E)=\left\{\varphi \in \Gamma(\dot{i}): H^{\Gamma}(\varphi) E_{\mid \operatorname{dom} \varphi} \subset E\right\} \text {, } \\
& A(E)=\left\{X \in T M: \varphi_{t} \in A(E) \text { where } \varphi_{t} \text { is the flow of } X\right\} \text {. }
\end{aligned}
$$

The bundle $E$ is called homogenous iff for each $x, y \in M$ there exists $\varphi \in A(E)$ such that $\varphi(x)=y ; \quad E$ is called tran sitive iff for each $z_{1}, z_{2} \in E$ there exists $\psi \in A(E)$ such that $\mathrm{H}^{\mathrm{r}}(\psi) \mathrm{z}_{1}=\mathrm{z}_{2}$. The bundle $\mathrm{E}$ is called infinitesimally homogenous iff for each $x \in M$ and each $v \in T_{x} M$ there exists $X \in A(E)$ such that $X_{x}=v ; \quad E$ is called infinitesimally tran sitive iff for each $z \in E$ and each $x^{r} \in T_{z} E$ there exists $x \in A(E)$ such that $H^{x}(x)_{2}=x^{r}$. Such meanings of (infinitesi mal) homogenity and (infinitesimal) transitivity are used for instance by P. Molino [5] .

Lemma III.5. If $E$ is a principal fibre bundle associa ted with the 0-deformable field of geometric objects $a$ then $A(\sigma)=A(E)$ and $A(\sigma)=A(E)$.

proof. It is enough to prove that $A(0)=A(E)$. 
Let $\varphi \in A(\sigma)$ then it is easy to notice that $\tau_{\sigma} \cdot \mathrm{H}^{r}(\varphi)=$

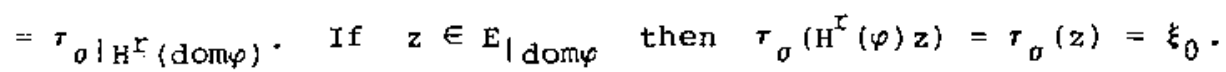
Hence $H^{\mathrm{T}}(\varphi) \mathrm{E}_{\text {! dom } \varphi} \subset \mathrm{E}$ and $\varphi \in \mathrm{A}(\mathrm{E})$.

On the other hand let $\psi \in A(E)$ and let $j_{0}^{r} f \in E_{x}$ where $x \in$ dom $\psi$. It implies that ${ }^{r}\left(H^{r}(\psi) j_{0}^{r}\right)=\tau_{u}\left(j_{0}^{x} f\right)=\xi_{0}$. From the definition of $\tau_{\sigma}$ we get that $F\left(f^{-1} \cdot \psi^{-1}\right) \sigma \psi(x)=$ $=F\left(f^{-1}\right) \sigma(x)$. Hence $F\left(\psi^{-1}\right) \sigma \psi(x)=\sigma(x)$ and $\psi \psi E A(\sigma)$. That ends the proof.

In the following proposition we compare (infinitesimal) homogenity and (infinitesimal) transitivity of the bundle $E$ and the field of geometric objects $\sigma$.

proposition III.6. If $\sigma$ is a 0 -deformable field of geometric objects and $\mathrm{E}$ is a principal fibre bundle generated by $\sigma$ then

i) $\sigma$ is homogenous iff $\mathrm{E}$ is homogenous;

ii) $\sigma$ is infinitesimally homogenous iff $E$ is infini tesimally homogenous;

iii) $\sigma$ is transitive iff $E$ is transitive;

iv) $\sigma$ is infinitesimally transitive iff $E$ is infini tesimally transitive.

Proof. The first condition is a simple consecjuence of Lemma III.5.; ii). one can easy get from lemma II.2. Similary iv). easly follows from lemma II.2. and lemma III.5.

To prove $i i i)$. it is enough to notice that if $z_{1}, z_{2} \in E$ and $\varphi \in \Gamma(M)$ then $\mathrm{H}^{\mathrm{r}}(\varphi) \mathrm{z}_{1}=z_{2}$ iff $\mathrm{j}_{\mathrm{x}^{\varphi}}^{r}=\mathrm{z}_{2} \cdot \mathrm{z}_{1}^{-1}$ where $\mathrm{x}$ 
is the source of $z_{1}$. Hence the third equivalence is an easy consequence of lemma II.1. and lemma III.5.

\section{REFERENCES}

1. J. Aczel und $\mathrm{S}$. Gołab: Funktionalgleichungen der Geometrischen objekte. PWN Warszawa 1960.

2. H. Goldschmidt, D.C.Spencer: on the non-linear cohomology of Lie equations.

I. Acta Math. 1976, 136 no 1-2 p. 103-170;

II. Acta Math. 1976, 136 № 3-4 p. 171-239;

III. J. Diff. Geom., 197813 no 3, p. 409-453;

IV. J. Diff. Geom., 197813 no 4, p. 455-526;

v. J. Diff. Geom., 1982 i6 no 4, p. 595-674.

3. A.K. Kumpera, D.C. Spencer: Lie equations: I general theory. Annals of Math. Studies № 73, Princeton Univ. Press, 1972.

4. B. Malgrange: Equations de Lie: I,II - J. Diff. Geom., 6, 1972, p. 503-522; 7, 1972, p. 117-141.

5. P. Molino: Theorie des G-structures: Le problem d'equivalen ce. Lecture Notes in Math, no 588 Springer Verlag 1977.

6. Ngo Van Que: Du prolongement des espaces fibrés et des structures infinitesimales. Ann. Inst, Fourier, Grenoble 
$24,1(1974), 297-310$.

7. A. Nijenhuis: Natural bundles and their general properties. Diff. Geom. in honour of $\mathrm{K}$. Yano Kinokuniya (1972).

8. P.S. Palais, C.L. Terng: Natural bundles have finite order. Topology 16 (1978) p. 271-277.

9. S.E. Salvioli: On the theory of geometric objects. J. Diff. Geom. (1972) P. 257-278.

10. A. Zajtz: Foundations of Differential Geometry on Natural Bundles. Caracas 1984 .

Rebut el 18 d'octubre del 1984

Instytut Matematyki

Uniwersytet Jagiellonski

U1. Reymonta 4, 30-059 Krakow

POLAND 